\title{
Electrical characterization of electrochemically grown single copper nanowires
}

\author{
M. E. Toimil Molares ${ }^{\text {a) }}$ \\ Gesellschaft für Schwerionenforschung (GSI), Planckstraße 1, D-64291, Darmstadt, Germany \\ E. M. Höhberger, Ch. Schaeflein, and R. H. Blick \\ Center for Nanoscience and Sektion Physik, Ludwig-Maximilians-Universität, Geschwister-Scholl-Platz, 1, \\ 80539 Munich, Germany \\ R. Neumann and C. Trautmann \\ Gesellschaft für Schwerionenforschung (GSI), Planckstraße 1, D-64291, Darmstadt, Germany
}

(Received 8 November 2002; accepted 3 February 2003)

\begin{abstract}
Single- and poly-crystalline copper wires with diameters down to $30 \mathrm{~nm}$ are grown in etched ion-track membranes. Individual nanowires are isolated and contacted by means of optical lithography. Electronic transport properties and oxidation processes are investigated. Depending on the oxidation state, the wire resistance varies between a few hundred ohms and several megaohms, enabling its usage as metallic or semiconducting structural elements for devices on the nanometer scale. (C) 2003 American Institute of Physics. [DOI: 10.1063/1.1563741]
\end{abstract}

Research on nanowires is continuously increasing since the range of possible applications is extremely broad, including optics, ${ }^{1}$ magnetism, ${ }^{2-4}$ and electronics. ${ }^{5}$ Fabrication and control of nanometer-sized conducting wires synthesized from a wide range of materials are crucial technological aspects with regard to contacting and operating nanoscale electronic systems. In particular, nanowires functioning as semiconductor elements or simply as connectors to or within molecular devices are very promising. ${ }^{5}$

A high flexibility in the design of nanowires regarding length, diameter, and aspect ratio is desirable for their application as connecting elements and provides, at the same time, a unique tool for investigating the extraordinary properties that these nanostructures exhibit due to their reduced size. Among the different techniques available for the fabrication of such wires, ${ }^{6-8}$ the so-called template method fulfills these demands. ${ }^{8-12}$ This method is based on the growth of wires in the pores of a matrix, commonly named a template. The technique allows the creation of wires of various lengths (up to $100 \mu \mathrm{m}$ ) and diameters (as small as a few nanometers), with a maximum aspect ratio of $10^{3}-10^{4}$. Etched ion-track membranes and $\mathrm{Al}_{2} \mathrm{O}_{3}$ structures are the predominantly employed templates. Electrochemical deposition in the pores provides high-quality nanowires of different metals with a very smooth surface. ${ }^{13}$

So far, electrical measurements have been performed on different kinds of nanowires; for example, $\mathrm{VnO}_{5},{ }^{14} \mathrm{Au},{ }^{15}$ $\mathrm{Mo},{ }^{6} \mathrm{Bi},{ }^{11}$ and several semiconductors. ${ }^{16,17}$ In all cases, it has been quite difficult to achieve a reliable contact with resistances of the order of only ohms. Several attempts have been made to determine the intrinsic properties of the wires and to distinguish them from external effects, such as adsorption of a contamination layer or oxidation of the wire. Electrical measurements on electrochemically grown nanowires were mostly performed on a large assembly of wires embedded in

${ }^{a)}$ Electronic mail: e.toimil@gsi.de a membrane. ${ }^{11,18,19}$ In these cases, the contacting technique did not allow to measure an absolute value of the resistance of a single wire.

Here, we report a method for producing an ensemble of single and polycrystalline $\mathrm{Cu}$ nanowires in polycarbonate etched ion-track membranes. We then describe how, after dissolving the membrane, single copper nanowires are individually contacted by means of optical lithography and characterized by transport measurements at $4.2 \mathrm{~K}$ and at room temperature. This technique combines the production and preparation of metallic nanowires, possessing a homogeneous and smooth contour of different crystallographic characteristics, with the flexibility and resolution of lithographic techniques.

The production of the wires includes the following steps, schematically depicted in Fig. 1(a). First, polycarbonate foils (Makrofol N, thickness $30 \mu \mathrm{m}$ ) are irradiated at the UNILAC linear accelerator of GSI, with ions of energy $11.4 \mathrm{MeV} /$ nucleon, applying fluences between $10^{6}$ and $10^{9}$ ions $/ \mathrm{cm}^{2}$. Along their trajectory through matter, the ions create cylindrical tracks of modified material. These tracks are chemi- (a)

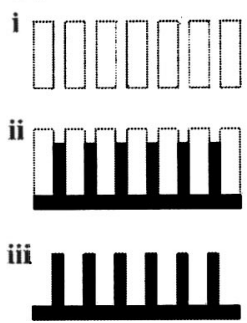

(b)
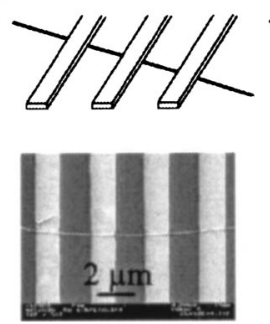

(c)
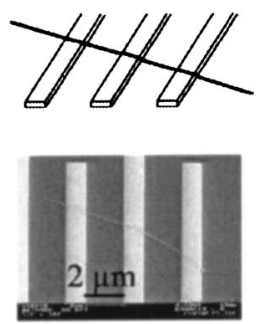

FIG. 1. (a) Process steps for electrodeposition: (i) a template is generated by chemical etching of the ion tracks; (ii) $\mathrm{Cu}$ is deposited in the pores; (iii) the supporting membrane is dissolved. (b), (c) Two different arrangements for attaching metallic contacts to $\mathrm{Cu}$ nanowires: preferably, the electrodes are fabricated on top of the nanowires (b), or the wires are deposited on the prefabricated contacts (c). 
cally etched in a solution of $6 \mathrm{~N} \mathrm{NaOH}$ containing 10 vol \% of methanol at $50{ }^{\circ} \mathrm{C}$, resulting in cylindrical pores. ${ }^{20}$ Depending on the etching time, pores with diameters between several nanometers and some micrometers can be produced [Fig. 1(a,i)]. A conductive layer is deposited on one side of the membrane (some $100 \mathrm{~nm}$ of sputtered $\mathrm{Au}$ and several micrometers of galvanostatically deposited $\mathrm{Cu}$ ) to serve as cathode and stable mechanical support for the subsequent potentiostatic growth of the $\mathrm{Cu}$ nanowires in the pores [Fig. 1(a,ii)]. The employed electrolyte consists of an aqueous solution of $238 \mathrm{~g} / \mathrm{L} \mathrm{CuSO}_{4} \cdot 5 \mathrm{H}_{2} \mathrm{O}$ and $21 \mathrm{~g} / \mathrm{L}$ sulfuric acid. By varying the deposition parameters (i.e., composition of the electrolyte, temperature, and deposition voltage) both single and polycrystalline wires were created. ${ }^{12}$

Before contacting the wires for electrical transport measurements, the polymer has to be eliminated. For this purpose, the template is dissolved in dichloromethane [Fig. 1(a,iii)]. The wires are then detached from the bottom by inserting the sample, placed in a beaker, in an ultrasonic bath, followed by centrifugation of the solution. Subsequently, the solution above the wires is exchanged with a pipette, and the beaker is again put into the ultrasonic bath. By repeating this procedure several times, the wires are cleaned efficiently.

Finally, in order to perform electrical measurements, wires are contacted using two different configurations: wires "below" and wires "above" the contacts. In the first case [below, Fig. 1(b)], some drops of dichloromethane solution containing the wires are first given on a $\mathrm{SiO}_{2}$ substrate. After evaporation of the solvent, an array of about 100 contacting structures, each consisting of four finger-like electrodes is created by means of optical lithography and metal evaporation on top of the wires. In the second case [above, Fig. 1(c)], the electrodes are deposited first, and the wires are subsequently placed on top. In both cases, the number of wires on the substrate is controlled by varying the concentration of wires in the solution and/or the number of drops deposited on the substrate. As indicated in Figs. 1(b) and 1(c), the four Au contacts usually have a width of $1 \mu \mathrm{m}$ and spacing of 2 $\mu \mathrm{m}$. After bonding the sample in a standard chip carrier, it is mounted in an evacuated chamber containing only a minute amount of He exchange gas that allows thermal equilibration. Transport measurements can then be performed in a straightforward two- (or four-) terminal geometry by applying a constant voltage to the nanowires and recording the current with picoamp resolution using an Ithaco 1211 current preamplifier. ${ }^{21}$ For measurements at $T=4.2 \mathrm{~K}$, the sample holder is inserted in liquid He.

Using the technique described in Fig. 1(c) (nanowires on top of the contacts), resistances of the order of gigaohms were recorded, possibly due to the existence of an adsorbate layer between the gold contacts and the wires. ${ }^{14}$ Hence, all presented measurements were performed using the favorable contacting scheme of Fig. 1(b), which also avoids mechanical deformation of the wires.

In Fig. 2(a), the $I-V$ characteristic of a single-crystalline $\mathrm{Cu}$ wire with a diameter of $60 \mathrm{~nm}$ is depicted. The micrograph shows a scanning electron microscope (SEM) image of the Au electrodes patterned on top of the wire. The length of the wire is of the order of $2.4 \mu \mathrm{m}$. The trace is ohmic, (a)
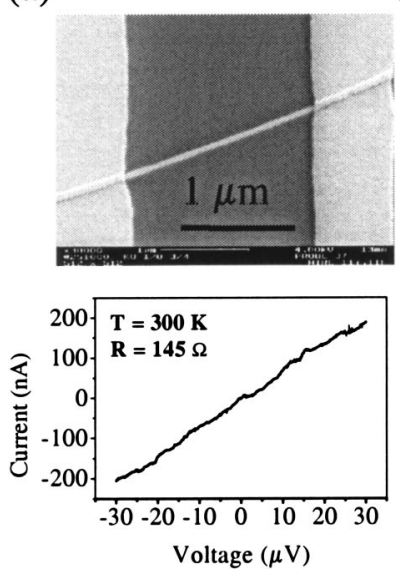

(b)
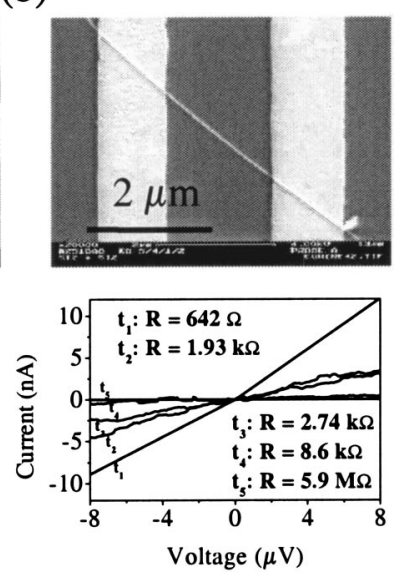

FIG. 2. (a) $I-V$ characteristic of a $60-\mathrm{nm}$-diameter $\mathrm{Cu}$ nanowire fabricated according to the method depicted in Fig. 1(b), clearly indicating ohmic behavior (SEM micrograph visualizes the contact pads and the nanowire). (b) Aging by oxidation of a nanowire: the resistance steadily increases the more $\mathrm{Cu}_{2} \mathrm{O}$ has developed (SEM image shows the actual wire orientation).

indicating metallic behavior with a resistance of $145 \Omega$ at room temperature. This gives a specific resistivity of $\rho$ $=1.71 \times 10^{-5} \Omega \mathrm{cm}$, which is about 10 times higher than that for bulk $\mathrm{Cu}$ at $300 \mathrm{~K}$. This enhanced value can be due to a high contact resistance, to surface scattering of the electrons within the nanowire, ${ }^{22}$ or to an onset of $\mathrm{Cu}$ oxidation. ${ }^{23,24}$ Since with the employed electrode configuration much smaller contact resistances are expected, we think that the two latter effects contribute to the measured values. This assumption is also supported by results described subsequently.

Figure 2(b) displays several $I-V$ curves of a $50-\mathrm{nm}$ diameter $\mathrm{Cu}$ nanowire recorded over $12 \mathrm{~h}$ at room temperature. Within this time period, the resistance gradually increases from the initial value of $640 \Omega$ by six orders of magnitude, documenting the ongoing oxidation subject to the permanent exposure to aerial oxygen. Since the oxidation process is expected to be enhanced due to the net temperature increase when the wire carries a large current, we attribute the tremendous growth of resistance to a complete oxidation of the wire. Moreover, the extremely high surface to volume ratio of the nanowires makes them more sensitive to oxidation. A similar effect has been reported for Mo nanowires. ${ }^{6}$ Figure 3 shows the $I-V$ characteristics at both room temperature and $4.2 \mathrm{~K}$ for the $\mathrm{Cu}$ wire depicted in Fig. 2(a) in its metallic state (a) and for the wire shown in Fig. 2(b) after complete oxidation (b). Prior to oxidation, the curves at 300 and $4.2 \mathrm{~K}$ are linear, and indicate a resistance drop by a factor of 1.5. After complete oxidation, the wire consists of $\mathrm{Cu}_{2} \mathrm{O}$ and should function as a $p$-type semiconductor. With the two electrodes, the $\mathrm{Cu}_{2} \mathrm{O}$ nanowire effectively creates two Schottky contacts in series placed back-toback. Hence, we observe a double-diode like $I-V$ characteristic $^{25}$ with temperature-activation behavior [see Fig. 3(b)]. The breakdown voltage at room temperature is about $\pm 2 \mathrm{~V}$. In the large bias regime up to $\pm 9 \mathrm{~V}$ [see Fig. $3(\mathrm{c})$ ], in which current densities of approximately $40 \mathrm{kA} / \mathrm{cm}^{2}$ are reached, the nanowire finally fails by breaking [see SEM micrograph in the inset of Fig. 3(c)]. 

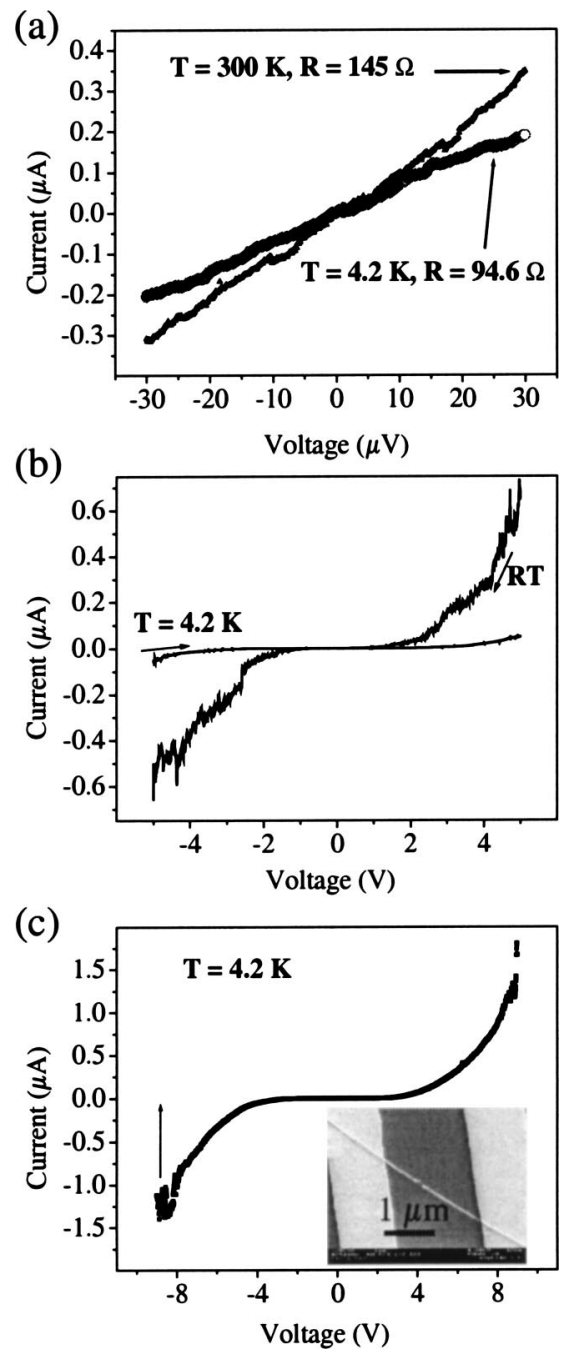

FIG. 3. (a) $I-V$ characteristic of the initial metallic $\mathrm{Cu}$ nanowire, depicted in Fig. 2(a), at $4.2 \mathrm{~K}$ and at room temperature: Both cases reveal ohmic behavior with slight increase in resistance with temperature. (b) $I-V$ curves of the wire shown in Fig. 2(b) for $4.2 \mathrm{~K}$ and room temperature recorded after oxidation. The previously metallic $\mathrm{Cu}$ nanowire transformed into a semiconducting $\mathrm{Cu}_{2} \mathrm{O}$ nanowire, forming two Schottky $p$-contacts in series. (c) High-voltage regime of the low-temperature curve from (b) giving evidence of the rupture of the wire. Inset: SEM micrograph of the wire after rupture due to repeated ramping to $\pm 9 \mathrm{~V}$.

In summary, we have demonstrated a reliable method for the production of electrodes on $\mathrm{Cu}$ nanowires exhibiting low ohmic contact resistances. We were able to monitor the oxidation process over many hours. This oxidation becomes vis- ible by the change of the nanowire from the metallic to the semiconducting regime, with the double-diode characteristic.

We would like to acknowledge financial support from the Bundesministerium für Forschung und Technologie and the Deutsche Forschungsgemeinschaft through grant DFGB1/487-3. We also thank Dr. T. Yamada for helpful discussions.

${ }^{1}$ C. A. Foss, G. L. Hornyak, J. A. Stocker, and C. R. Martin, Adv. Mater. 5, 135 (1993).

${ }^{2}$ J. Rivas, A. Kazadi Mukenga Bantu, G. Zaragoza, M. C. Blanco, and M. A. López Quintela, J. Magn. Magn. Mater. 249, 220 (2002).

${ }^{3}$ L. Piraux, J. M. George, J. F. Despres, C. Leroy, E. Ferain, R. Legras, K. Ounadjela, and A. Fert, Appl. Phys. Lett. 65, 2484 (1994).

${ }^{4}$ A. Blondel, J. P. Meier, B. Doudin, and J.-Ph. Ansermet, Appl. Phys. Lett. 65, 3019 (1994).

${ }^{5}$ X. Duan, Y. Huang, Y. Cui, J. Wang, and C. M. Lieber, Nature (London) 409, 66 (2001).

${ }^{6}$ M. P. Zach, K. H. Ng, and R. M. Penner, Science 290, 2120 (2000).

${ }^{7}$ A. M. Morales and C. M. Lieber, Science 279, 208 (1998).

${ }^{8}$ C. R. Martin, Science 266, 1961 (1993).

${ }^{9}$ J. Vetter and R. Spohr, Nucl. Instrum. Methods Phys. Res. B 79, 691 (1993).

${ }^{10}$ P. A. Smith, C. D. Nordquist, T. N. Jackson, T. S. Mayer, B. R. Martin, J. Mbindyo, and T. E. Mallouk, Appl. Phys. Lett. 77, 1399 (2000).

${ }^{11}$ Z. Zhang, X. Sun, M. S. Dresselhaus, J. Y. Ying, and J. Heremans, Phys. Rev. B 61, 4850 (2000).

${ }^{12}$ M. E. Toimil Molares, V. Buschmann, D. Dobrev, R. Neumann, R. Scholz, I. U. Schuchert, and J. Vetter, Adv. Mater. 13, 62 (2001).

${ }^{13}$ M. E. Toimil Molares, J. Brötz, V. Buschmann, D. Dobrev, R. Neumann, R. Scholz, I. U. Schuchert, C. Trautmann, and J. Vetter, Nucl. Instrum. Methods Phys. Res. B 185, 192 (2001)

${ }^{14}$ J. Muster, G. T. Kim, V. Krstic, J. G. Park, Y. W. Park, S. Roth, and M. Burghard, Adv. Mater. 12, 420 (2000).

${ }^{15}$ P. A. Smith, C. D. Nordquist, T. N. Jackson, T. S. Mayer, B. R. Martin, J. Mbindyo, and T. E. Mallouk, Appl. Phys. Lett. 77, 1399 (2000).

${ }^{16}$ D. J. Pena, B. Razavi, P. A. Smith, M. J. Natan, T. S. Mayer, T. E. Mallouk, and C. D. Keating, Mater. Res. Soc. Symp. Proc. 636, D4.6.1 (2001).

${ }^{17}$ N. I. Kovtyukhova, B. R. Martin, J. K. N. Mbindyo, T. E. Mallouk, M. Cabassi, and T. S. Mayer, Mater. Sci. Eng., C 19, 255 (2002).

${ }^{18}$ A. Bachtold, C. Terrier, M. Krüger, M. Henny, T. Hoss, C. Strunk, R. Huber, H. Birk, U. Staufer, and C. Schönenberger, Microelectron. Eng. 41/42, 571 (1998).

${ }^{19}$ K. Liu, Cl. L. Chien, and P. C. Searson, Phys. Rev. B 58, 14681 (1998).

${ }^{20}$ R. L. Fleischer, P. B. Price, and R. M. Walker, Nuclear Tracks in Solids: Principles and Applications (University of California Press, Berkeley, 1975).

${ }^{21}$ Measurements shown here were performed only in a two-point geometry.

${ }^{22}$ C. Durkan and M. E. Welland, Phys. Rev. B 61, 14215 (2000).

${ }^{23}$ J. Li, J. W. Mayer, and E. G. Colgan, J. Appl. Phys. 70, 2820 (1991).

${ }^{24}$ Gmelin, Handbuch der Anorganischen Chemie, Chap. 60, Teil D (Verlag Chemie, Weinheim, 1955).

${ }^{25}$ S. M. Sze, Physics of Semiconductor Devices (Wiley, New York, 1981). 